\title{
Biología Reproductiva y Citogenética de Distichia muscoides (JUNCACEAE)
}

\author{
PAÚL GONZÁLES ${ }^{1}$, MERY SUNI ${ }^{2,3}$, ROCÍO DEANNA ${ }^{4,5}$, MARISEL A. SCALDAFERRO ${ }^{4,6}$, \\ ELENA CASTAÑEDA ${ }^{7}$, DÁMASO W. RAMIREZ ${ }^{1,8}$, NIELS VALENCIA ${ }^{3,9}$ y ASUNCIÓN CANO A $^{1,3}$
}

\begin{abstract}
Summary: Reproductive biology and cytogenetics of Distichia muscoides (Juncaceae). The genus Distichia Nees \& Meyen (Juncaceae) has three species from South America, and the most widely distributed is Distichia muscoides Nees \& Meyen. This dioecious species is the main component of the wetlands, an important ecosystem of the high Andean region. In this work, different biological aspects of $D$. muscoides were studied: 1) pollen viability through indirect staining techniques; 2) the stigmatic receptivity through hydrogen peroxide test; 3) the viability of the seeds; and 4) cytogenetic studies using fluorescent chromosome banding CMA/DA/DAPI. The results show that staminate flowers of $D$. muscoides have pollen with a high percentage of viability $(100 \%)$; pistillate flowers reach the stigmatic receptivity once stigma branches develop their maximum length $(4 \mathrm{~mm})$ and remain in this state for at least two days. The number of ovules per ovary is 34 , and the number of seeds per fruit is 25 . The seeds germinated since day 15 , showing $92 \%$ of viability and, an average on germination time of 18.4 days. The species has $2 n=80-84$ chromosomes and the fluorescent banding showed two pairs of terminal bands $\mathrm{CMA}^{+} / \mathrm{DAPI}$; associated with nucleolar organizer regions (NORs). Karyological and reproductive data for this genus are here reported for the first time.
\end{abstract}

Key words: Seed viability, dioecy, stigmatic receptivity, chromosome number, fluorescent banding, Juncaceae.

Resumen: El género Distichia Nees \& Meyen (Juncaceae) está formado por tres especies nativas de Sudamérica, siendo Distichia muscoides Nees \& Meyen la más ampliamente distribuida (Kirschner et al., 2002). Esta especie dioica es el componente principal de los bofedales, ecosistema muy importante de la región altoandina. En este trabajo se estudiaron distintos aspectos biológicos de $D$. muscoides como: 1) la viabilidad del polen por medio de métodos indirectos de coloración; 2) la receptividad estigmática a través de una prueba con peróxido de hidrógeno; 3) la viabilidad de las semillas mediante su germinación; y 4) sus datos citogenéticos, empleando la técnica de bandeo cromosómico fluorescente CMA/DA/DAPI. Los resultados muestran que las flores estaminadas presentan polen con un porcentaje alto de viabilidad (100\%); las flores pistiladas alcanzan la receptividad estigmática una vez que las ramas del estigma desarrollan su máxima longitud $(4 \mathrm{~mm})$ y permanecen en este estado al menos durante dos días. El número promedio de óvulos por ovario es 34 , y el número promedio de semillas por fruto es 25 . Las semillas germinaron a partir del día 15, mostrando $92 \%$ de germinación y un tiempo promedio de germinación de 18,4 días. La especie posee $2 n=80-84$ cromosomas y el bandeo de fluorescencia mostró dos pares de bandas terminales $\mathrm{CMA}^{+} / \mathrm{DAPI}$, asociadas a regiones organizadoras nucleolares (NORs). Se reportan por primera vez datos reproductivos y cariológicos para esta especie.

Palabras clave: Viabilidad de semillas, dioecia, receptividad estigmática, número cromosómico, bandeo de fluorescencia, Juncaceae.

\footnotetext{
1Laboratorio de florística, Departamento de Dicotiledóneas, Museo de Historia Natural, Universidad Nacional Mayor de San Marcos (UNMSM), Av. Arenales 1256, Lima, Perú. E-mail: pgonzalesarce@hotmail.com

${ }^{2}$ Laboratorio de Fisiología Vegetal, Facultad de Ciencias Biológicas (UNMSM), Lima, Perú.

${ }^{3}$ Instituto de Investigación de Ciencias Biológicas Antonio Raimondi, Facultad de Ciencias Biológicas (UNMSM), Lima, Perú.

${ }^{4}$ Instituto Multidisciplinario de Biología Vegetal (CONICET-UNC), Casilla Postal 495, 5000, Córdoba, Argentina.

${ }^{5}$ Facultad de Ciencias Químicas (UNC), Córdoba, Argentina.

${ }^{6}$ Facultad de Ciencias Exactas, Físicas y Naturales (UNC), Córdoba, Argentina.

${ }^{7}$ Facultad de Ciencias Biológicas (UNMSM), Lima, Perú.

${ }^{8}$ Área de Ecología, Cursos Básicos de Ciencias, Universidad Científica del Sur, Lima, Perú.

${ }^{9}$ Dep. Ecología, Museo de Historia Natural (UNMSM), Lima, Perú.
} 


\section{INTRODUCCIÓN}

La familia Juncaceae es probablemente la menos estudiada dentro del orden Poales en cuanto a estudios de reproducción, anatomía y citología (Záveská Drábková, 2013). Esta familia alberga 450 especies en ocho géneros y tiene una amplia distribución en las regiones templadas y árticas de ambos hemisferios. En los Andes Sudamericanos son nativos los géneros Distichia Nees \& Meyen, Patosia Buchenau y Oxychloe Phil., creciendo en forma de almohadillados.

Distichia muscoides Nees \& Meyen es una planta dioica que forma almohadillados densos y duros de varios metros de diámetro. Se encuentra ampliamente distribuida desde Colombia hasta Argentina entre los 3800 y 4600 m s.n.m. (Balslev, 1996; Kirschner et al., 2002). Se caracteriza por ser el componente principal de los bofedales, formaciones vegetales hidromórficas que se desarrollan en fondos de quebradas, pendientes o en planicies altoandinas (Valencia et al., 2013). Distichia muscoides puede formar islas flotantes en suelos pantanosos, colmatar cuerpos de agua o crecer en las márgenes de los arroyos. El hábitat "bofedales" en donde se desarrolla D. muscoides tienen un rol importante en la regulación del balance hídrico a nivel regional y local (Weberbauer, 1945; Tovar, 1973). Además, estos ecosistemas son considerados sensibles, debido a su alta fragilidad y a las amenazas que le aquejan, por ejemplo la extracción de D. muscoides "turba" para uso como combustible (Ferreyra, 1979).

Se piensa que la principal forma de reproducción de estas plantas es la asexual, donde las raíces de Distichia se forman a lo largo de los tallos ascendentes, penetrando la cubierta densa de vainas foliares, desarrollando ramas nuevas de igual grosor que la rama principal (Ruthsatz, 1978). En cuanto a su periodo reproductivo, la floración empieza en agosto y termina en noviembre; los primeros frutos inmaduros se pueden observar a inicios de enero, mientras que los maduros se observan hacia fines de mayo, aparentemente este ciclo sería temporalmente irregular pudiendo repetirse anualmente o cada dos o tres años (D. W. Ramírez, com. pers.). Estas plantas son dioicas con flores "unisexuales" y solitarias, las cuales se originan en la axila de una hoja del tallo. Las flores pistiladas de Distichia nacen en pedúnculos cortos, y sólo los estigmas receptivos se proyectan por encima del ápice del brote y el denso almohadillado. En contraste, las flores estaminadas de Distichia nacen en pedúnculos largos elevados por encima del almohadillado (Balslev, 1996; Kirschner et al., 2002).

El polen en la familia Juncaceae permanece unido en tétradas, rodeado de una capa común derivada de las células madre del polen denominado esporocine (Wille, 1882, citado por Balslev, 1996). Los granos de polen de Distichia y sus parientes cercanos (Oxycloe y Patosia) son relativamente más grandes que los del género Juncus L., lo que indicaría una mayor adaptación a la anemofilia de este último género (Balslev, 1996).

Dentro de Juncaceae se han realizado estudios citogenéticos tradicionales o mediante tinción fluorescente, principalmente en Juncus L. ( $\mathrm{x}=$ 20) y Luzula DC. $(\mathrm{x}=6)$, aunque el $58 \%$ de sus taxones permanece citogenéticamente desconocido (Záveská Drábková, 2013). Los complementos cromosómicos de las especies pertenecientes a Juncaceae usualmente forman series poliploides, siendo los números más comunes correspondientes a especies diploides: $2 \mathrm{n}=12$ y $2 \mathrm{n}=40$ (Záveská Drábková, 2013). También se ha reportado aneuploidía en Oxychloe, género más emparentado con Distichia (Balslev, 1996; Záveská Drábková, 2013), y se ha observado la presencia de centrómero difuso en algunos géneros de la familia (Bozek et al., 2012; Bureš et al., 2013). Distichia y Patosia Buchenau son dos géneros de la familia Juncaceae hasta el momento cariológicamente desconocidos.

El presente trabajo tiene como objetivo evaluar distintos aspectos de la biología reproductiva y citogenética de D. muscoides, tales como la viabilidad del polen, la receptividad estigmática, la viabilidad de las semillas, el rol del agua como vector de polen y la citogenética, reportando la primera aproximación de su número cromosómico. Se abordan estos aspectos a fin de indagar los posibles efectos de estos atributos en la eficiencia reproductiva.

\section{Materiales y Métodos}

\section{Estudios reproductivos}

El material proviene de la zona de Rosaspata (Distrito de Vinchos, Prov. Huamanga, Dpto. 


\section{P. Gonzáles et al. - Biología reproductiva y citogenética de Distichia muscoides}

Ayacucho, Perú) a 4500 m s.n.m. (Fig. 1A). Los sitios de colección fueron visitados entre el 17-18 de octubre del 2014 (voucher Gonzáles et al. 3400a, planta femenina, 3400b, planta masculina; USM). Se colectó material de 15 y $25 \mathrm{~cm}$ de diámetro "champas" provenientes de cuatro almohadillados que se encontraban a inicio de floración (dos plantas masculinas y dos femeninas separadas por más de 50 metros). Las plantas fueron aclimatadas en una cámara de flujo laminar con fotoperiodo $12: 12$ y termoperiodo de $16^{\circ} \mathrm{C}$ en la noche y $25^{\circ} \mathrm{C}$ en el día, durante ocho días.

Se realizó una caracterización de las flores masculinas y femeninas, donde se cuantificaron las dimensiones de cada atributo vinculado con la reproducción sexual de la especie (Tabla 1). Para el caso de las flores masculinas, se tomaron las medidas de cinco flores luego de la antesis. La viabilidad del polen fue evaluada mediante el método indirecto de coloración. Se realizó la tinción con carmín acético, asumiendo a los granos de polen fuertemente teñidos como viables y a los de tinción baja o no teñidos como no viables. Se evaluó en 10 campos, los cuales fueron observados con microscopio óptico a 40X de aumento.

Para la caracterización morfológica de los granos de polen, se realizaron observaciones con microscopio óptico a 40X. Para cada carácter estudiado se menciona la dimensión mínima y máxima encontradas para el diámetro ecuatorial (DE), eje polar (EP), incluyendo la exina y diámetro de poros. Se tomaron como mínimo 5 mediciones. Las fotos fueron tomadas con cámaras de un microscopio digital Leica ICC50 y cámaras manuales digitales Canon PowerShot SX110 IS (Universidad Nacional Mayor de San Marcos, Perú). La terminología utilizada en la descripción polínica fue tomada de Faegri \& Iversen (1964), Heusser \& Moar (1973) y Hesse et al. (2009).

Para evaluar la receptividad estigmática, se establecieron cuatros estadios de las flores femeninas de $D$. muscoides tomando como parámetro la longitud del pistilo (Tabla 1; Fig. 2). La receptividad de los estigmas se detectó a través de la prueba de peróxido de hidrógeno (Galen \& Plowright, 1987). Fueron extraídos los estilos con la precaución de no dañar el estigma para evitar falsos positivos; los mismos fueron montados sobre un porta-objetos donde se le agregó una gota de peróxido de hidrógeno $3 \%$, considerando como receptivos aquellos estigmas que presentaban burbujeo. Para cada estadio, se realizaron cinco repeticiones.

La viabilidad de las semillas se estimó como el porcentaje de germinación. Se utilizaron 228 semillas que fueron desinfectadas con hipoclorito de sodio al $2,6 \%$ por 30 min y se enjuagaron tres veces con agua destilada estéril. Se sembraron en

Tabla 1. Dimensiones de los atributos florales de las flores masculinas adultas (indicando dimensiones del gineceo reducido) y en cuatro estadios de flores femeninas de Distichia muscoides indicando la receptividad correspondiente.

\begin{tabular}{|c|c|c|c|c|c|}
\hline \multirow{2}{*}{ Características } & \multirow{2}{*}{$\begin{array}{c}\text { Flores } \\
\text { masculinas }\end{array}$} & \multicolumn{4}{|c|}{ Flores femeninas } \\
\hline & & Estadio 1 & Estadio 2 & Estadio 3 & Estadio 4 \\
\hline$N^{\circ}$ de óvulos & & 35 & 30 & 39 & 32 \\
\hline Receptividad/ Lugar & & Nula & Baja y apical & Alta y general & No receptivo \\
\hline Largo de la flor (mm) & $0,75-0,95$ & 4,19 & 7,42 & 11,64 & 11,04 \\
\hline Largo del Ovario (mm) & $0,20-0,21$ & 0,775 & 1,62 & 2,72 & 3,1 \\
\hline Ancho del Ovario (mm) & $0,17-0,23$ & 0,31 & 0,874 & 1,3 & 1,4 \\
\hline Largo del estilo (mm) & $0,13-0,16$ & 1,39 & 1,99 & 4,08 & 4,07 \\
\hline Largo de las ramas estigmáticas (mm) & $0,26-0,43$ & 2,2 & 4,02 & 4,85 & 4,8 \\
\hline Color de las ramas estigmáticas & blanquecinas & blanquecinas & blanquecinas & rojizo & Marrón \\
\hline Largo del filamento & $0,16-0,17$ & & & & \\
\hline Largo de anteras & $1,67-2,59$ & & & & \\
\hline Largo del mucrón & $0,11-0,12$ & & & & \\
\hline
\end{tabular}


Bol. Soc. Argent. Bot. 51 (1) 2016

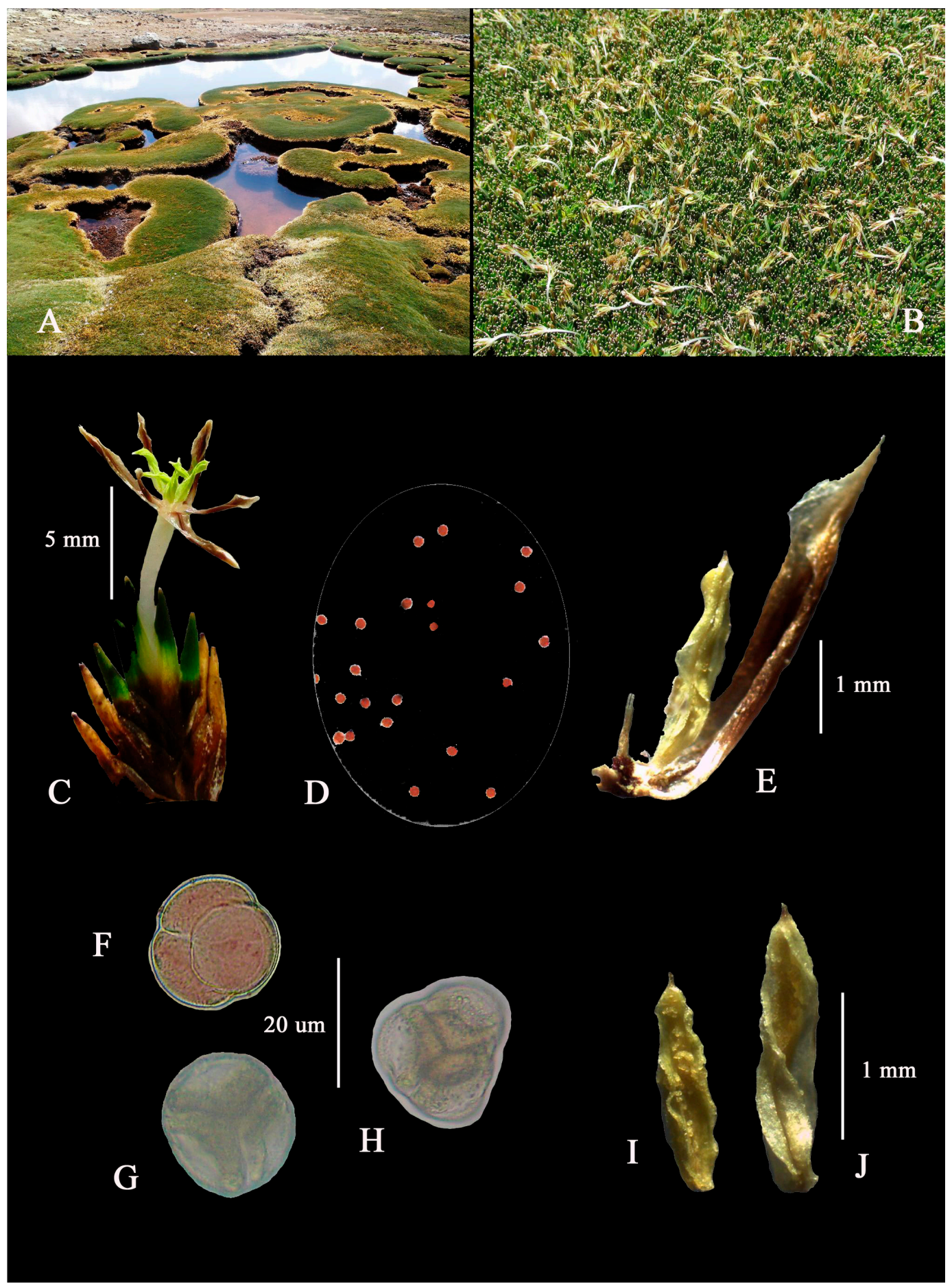

Fig. 1. A: Hábitat bofedal. B: Planta masculina. C: Flor estaminada. D: Viabilidad del polen, 40x. E: Porción de la flor estaminada mostrando un tépalo externo, un estambre y el gineceo reducido. F: Vista lateral del polen bajo tinción con carmín acético. G-H: Vista general del polen sin tinción. I: Estambre del verticilo interno. J: Estambre del verticilo externo. 


\section{P. Gonzáles et al. - Biología reproductiva y citogenética de Distichia muscoides}

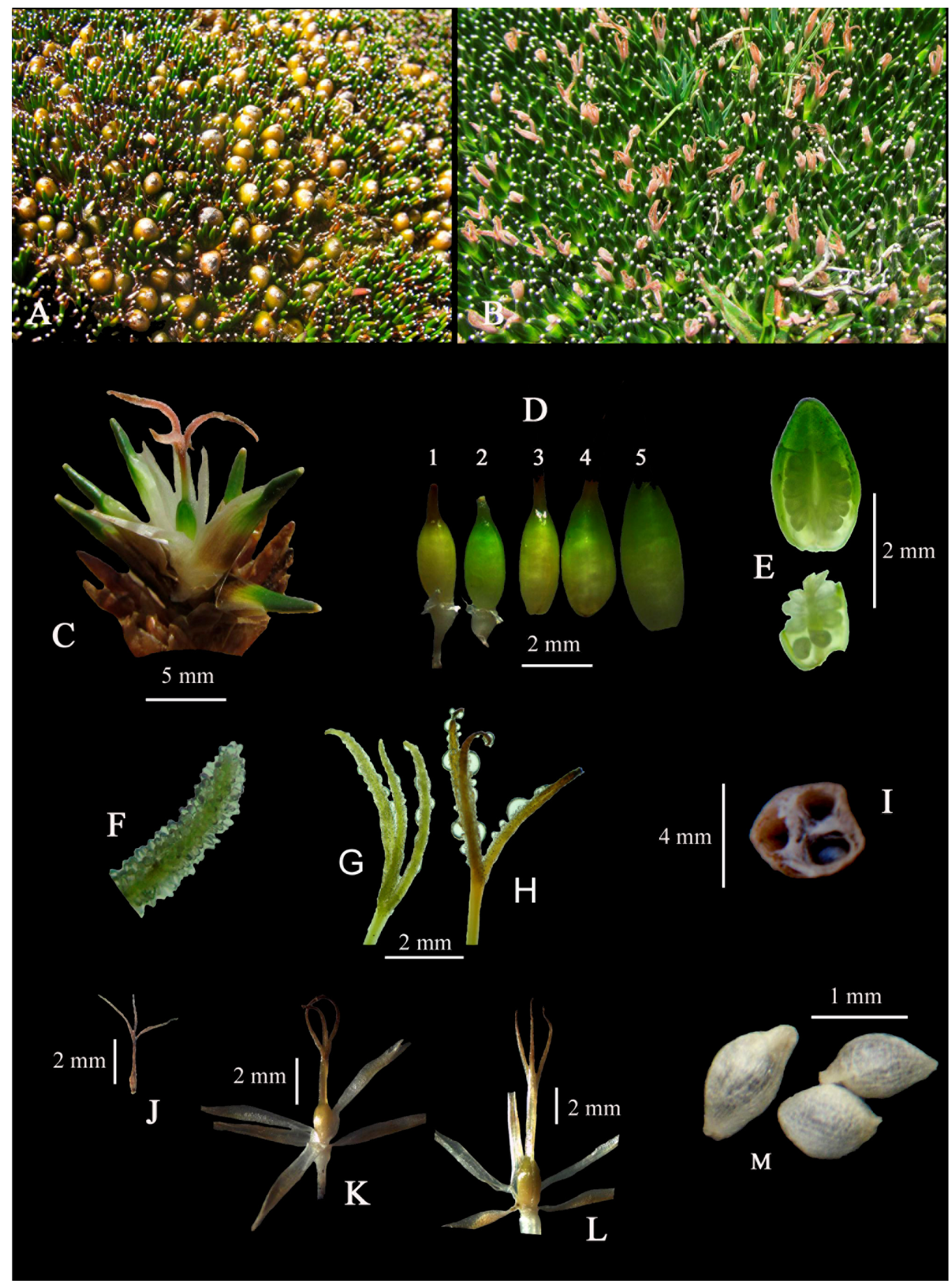

Fig. 2. A: Planta femenina en fructificación. B: Planta femenina en floración. C: Flor pistilada. D: Desarrollo del ovario (1 y 2: segundo estadio; 3: tercer estadio; 4 y 5: cuarto estadio). E: Sección longitudinal del ovario en el segundo estadio. F: Ampliación del ápice de las ramas estigmáticas papilosas. G-H: Receptividad estigmática positiva a la prueba del peróxido de hidrógeno (G. estadio 2; H. estadio 3). I: Sección transversal del fruto. J: Flor en estadio 1. K: Flor en estadio 2. L: Flor en estadio 3. M: Semillas. 
placas de Petri con papel de filtro húmedo con agua destilada, para luego colocarlas en una cámara de germinación con fotoperiodo $16: 8$ a $28^{\circ} \mathrm{C}$; las placas fueron regadas periódicamente a fin de mantener constantes las condiciones de humedad. Se consideraron germinadas a las semillas con radícula emergente mayor a $0,5 \mathrm{~mm}$. Las placas fueron revisadas cada cuatro días, retirando las semillas germinadas; el recuento de semillas germinadas se llevó de 15 a 30 días después de haber sido sembradas. Esta evaluación fue realizada entre los meses de noviembre y diciembre de 2014 en el Instituto Multidisciplinario de Biología Vegetal. Las semillas utilizadas fueron colectadas en campo el 20 de mayo del 2013, procedentes del departamento de Cusco-Perú (voucher Gonzáles et al. 2567; USM).

La participación del agua como vector de polen fue evaluado mediante el número de semillas producidas por fruto, que corresponde al número de óvulos fertilizados, asumiendo que a mayor oferta de polen por acción del agua, brinda mayor número de óvulos fertilizados. Para ello se diseñó un sistema de colecta de frutos donde se buscaron almohadillados que tengan una parte constantemente sumergida en el agua y otra no. Se colectaron frutos de 5 almohadillados considerando estos dos estratos. Además se colectaron semillas al azar en todo el área del almohadillado para considerarlo como control. Se analizaron 30 frutos por estrato y se aplicó el análisis de varianza (ANOVA) con el programa Past (Hammer et al., 2001).

\section{Estudios cariológicos}

Se utilizaron ápices radicales de semillas colectadas en el Dpto. Cusco-Perú (voucher Gonzáles et al. 2567, USM). Para la obtención de preparados cromosómicos se utilizaron raíces pre-tratadas con una solución saturada de paradiclorobenceno a temperatura ambiente y oscuridad durante $2 \mathrm{~h}$. Se fijaron en solución Farmer (alcohol etílico: ácido acético, $3: 1)$ y se conservaron a $4^{\circ} \mathrm{C}$ durante las primeras 24 horas, y luego a $-20^{\circ} \mathrm{C}$ hasta su uso. Se realizaron las preparaciones cromosómicas mediante digestión enzimática de las raicillas con celulasa $2 \%$ y pectinasa $1 \%$ a $37^{\circ} \mathrm{C}$ durante $150 \mathrm{~min}$ (Schwarzacher et al., 1980). Luego, los ápices radicales fueron aplastados con ácido acético $60 \%$ y se retiró el cubreobjeto mediante congelamiento con $\mathrm{CO}_{2}$ líquido (Bowen,
1956). Finalmente, los preparados se secaron a temperatura ambiente durante tres días. El bandeo se realizó mediante tinción triple (CDD) con los fluorocromos cromomicina $\mathrm{A} 3$, distamicina $\mathrm{A}$ y 4'-6-diamidino-2-fenilindol (CMA/DA/DAPI) según Schweizer (1980) y Schweizer \& Ambros (1994) con pequeñas modificaciones.

\section{Resultados}

\section{Estudios reproductivos}

Se caracterizaron las flores estaminadas maduras de $D$. muscoides cuyo resultado se muestran en la Tabla 1. En todas las flores examinadas se encontró un gineceo rudimentario menor a $1 \mathrm{~mm}$ de largo (Fig. $1 \mathrm{E}$ ).

Los granos de polen de D. muscoides están organizados en tétradas tetraédricas de 20,3-22,6 $\mu \mathrm{m}$ de diámetro ecuatorial y 20,0-22,1 $\mu \mathrm{m}$ de diámetro polar con exina tectada, psilada, de $1 \mu \mathrm{m}$. La exina es delgada y aparentemente desaparece donde se tocan los cuatro granos; es lisa pero con pequeños gránulos en los bordes de los granos y las suturas entre los cuatro granos son visibles a través de él (Fig. 1 F-H). La viabilidad del polen fue muy alta observando todos los granos de polen fuertemente teñidos, de forma y tamaño similar, indicando un $100 \%$ de viabilidad (Fig. 1 D).

Los estigmas se presentaron receptivos solo en los estadios dos y tres (Tabla 1; Fig. 2 G-H) aunque en el estadio tercero avanzado, se evidenció la presencia de un mayor burbujeo (Fig. 2 G). En el primer y cuarto estadio no mostró una reacción positiva con el peróxido de hidrógeno.

Las flores pistiladas del primer estadio son $3 \mathrm{~mm}$ más pequeñas que en el segundo, y en este último a su vez son $3 \mathrm{~mm}$ más pequeñas que en el tercero, mientras que este estadio no se diferencia del cuarto. Las diferencias en los tamaños de las flores pistiladas del segundo y tercer estadio, se deben principalmente al grado de desarrollo del estilo y del ovario, ya que las ramas estigmáticas en ambos estadios son de tamaño similar (Tabla 1, Fig. 2). A pesar de que las ramas estigmáticas manifestaron diferencias de coloración, en todos los estadios se evidenció la presencia de abundantes papilas translúcidas, principalmente en la cara adaxial. En el cuarto estadio, (Fig. 2 D4-5) el ovario tiene mayor tamaño que en los estadios anteriores. 
El porcentaje de germinación de las semillas de D. muscoides resultó muy elevado (92\%), entre los 15 y 30 días luego de haber sido sembradas (Tabla 2). Cabe resaltar que cerca del $82 \%$ germinaron en los primeros 19 días, obteniéndose un tiempo promedio de germinación de 18,4 días.

Respecto a la participación del agua como vector de polen, el promedio del número de semillas para el tratamiento control fue de 27, correspondiendo 25 para la parte expuesta y 26 para la parte sumergida (Fig. 4). El análisis de varianza indicó que no hay diferencias significativas entre los diferentes tratamientos $(\mathrm{p}=0,8949)$. El número promedio de óvulos por ovario es $34 \pm 3,916$ con un mínimo de 25 y un máximo de 40 , y el número promedio de semillas por fruto es $25 \pm 4,835$ con un mínimo de 10 y un máximo de 40 (éxito reproductivo de 75\%).

\section{Estudios cariológicos}

Los recuentos cromosómicos resultaron dificultosos debido a la presencia de numerosos cromosomas, pudiéndose estimar que su número se encuentra entre $2 n=80$ y $2 n=84$. Mediante la técnica de bandeo cromosómico CMA/DA/ DAPI, se detectaron 4 bandas $\mathrm{CMA}^{+} / \mathrm{DAPI}^{-}$ en el complemento cromosómico, asociadas a regiones organizadoras nucleolares (NORs). Un par de bandas se observó en dos cromosomas metacéntricos $(\mathrm{m})$ de mayor tamaño, mientras que el segundo par se asoció a dos cromosomas submetacéntricos (sm) de menor tamaño (Fig. 3). La presencia de cromosomas con la morfología descripta ( $\mathrm{m}$ y $\mathrm{sm}$ ), denota la existencia de una constricción primaria.

Tabla 2. Número de semillas germinadas de Distichia muscoides según los días después de sembrarlas.

\begin{tabular}{|cc|}
\hline Días después del sembrado & Semillas germinadas \\
\hline 15 & 71 \\
19 & 111 \\
23 & 18 \\
27 & 11 \\
31 & 0 \\
\hline Total de semillas germinadas & 211 \\
Semillas sin germinar & 17 \\
\hline
\end{tabular}

\section{Discusión}

\section{Estudios reproductivos}

Todas las dimensiones obtenidas en las flores estaminadas y pistiladas se encuentran dentro del rango de variación de las especies reportadas por Balslev (1996) y por Kirschner et al. (2002). En este trabajo, se reporta por primera vez la existencia
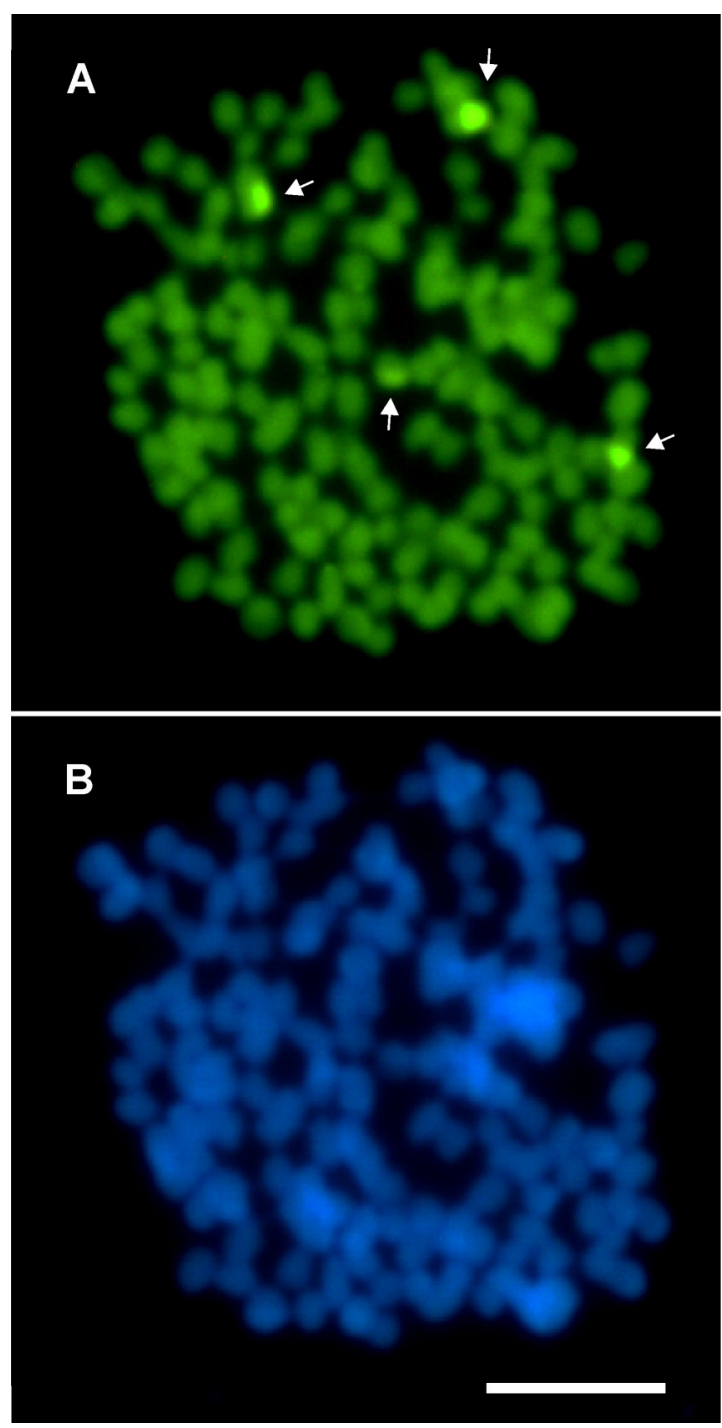

Fig. 3. Cromosomas metafásicos de $D$. muscoides con bandeo cromosómico fluorescente CMA/DA/ DAPI. A. Bandeo CMA. B. Bandeo DAPI. Las flechas indican las bandas $\mathrm{CMA}^{+} / \mathrm{DAPI}^{-}$y la escala representa $5 \mu \mathrm{m}$. 


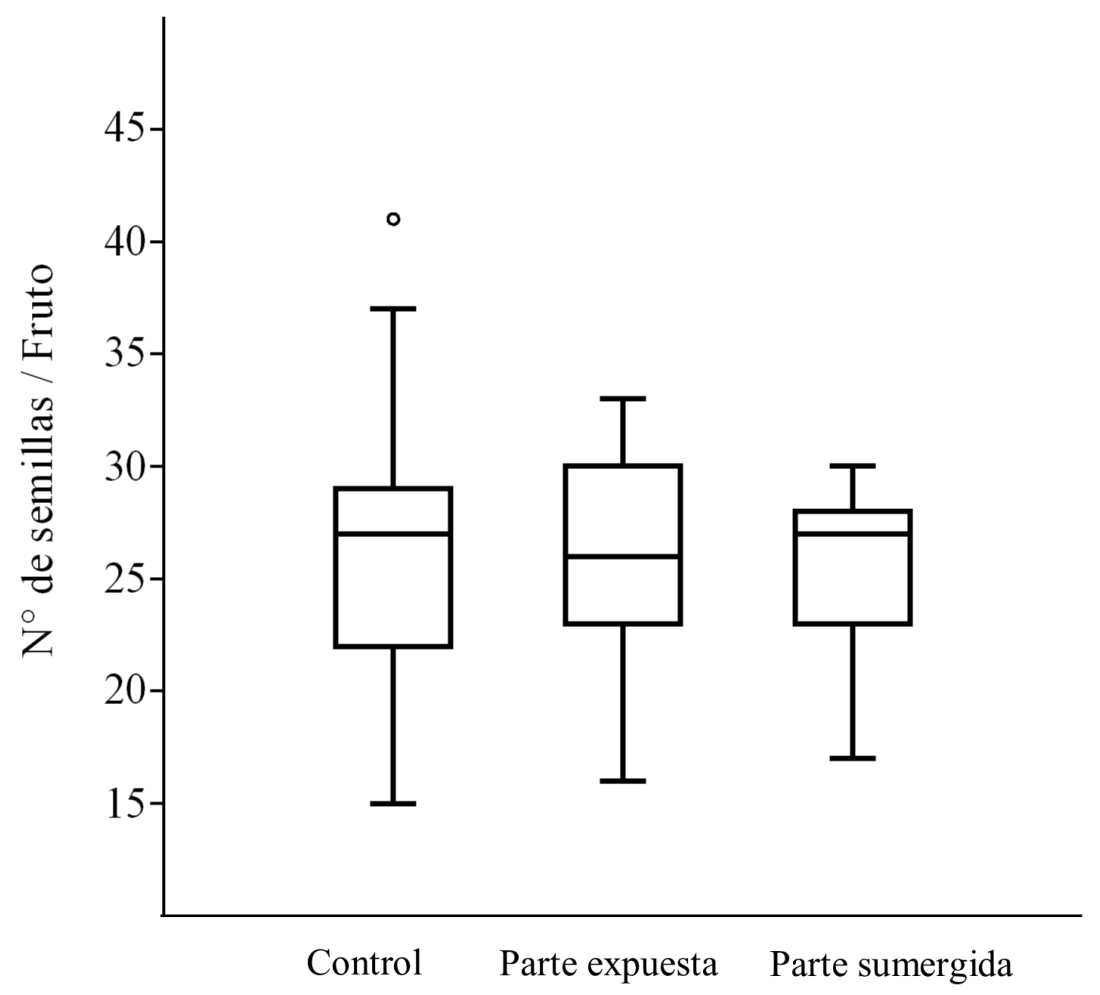

Fig. 4. Número de semillas promedio por fruto en los distintos tratamientos.

de un pistilodio o gineceo rudimentario en las flores estaminadas de 0,7-1,0 mm de largo, que solo había sido reportado para $D$. acicularis con un tamaño de 1,5 mm de largo (Balslev, 1996; Kirschner et al., 2002), pudiendo ser un carácter constante en todas las especies del género (D. W. Ramírez, com. per.). Estos tipos de flores representarían un estado transitorio en la evolución a la unisexualidad.

La forma tetraédrica del polen de $D$. muscoides es muy particular y permite diferenciarlas a este nivel de otros grupos taxonómicos (polen en monadas) que habitan turberas (Torres et al., 2012), exceptuando los de Oxychloe y Patosia quienes probablemente tengan una conformación similar. En el presente trabajo se reporta el polen de D. muscoides, de 20-23 $\mu \mathrm{m}$ de diámetro. Estos resultados no concuerdan con los registrado por Torres et al. (2012) para esta misma especie proveniente de las turberas altoandinas de Jujuy, cuyas dimensiones oscilan entre los 50-52 $\mu \mathrm{m}$. La exina en $D$. muscoides es delgada, cuya adaptación está vinculada con la anemofilia, que es el modo dominante de la polinización en Juncaceae
(Balslev, 1996).

En el segundo y tercer estadio el estigma se encontró receptivo, sin embargo en el tercer estadio se observó una mayor cantidad de burbujeo sobre el estigma que podría asociarse a una mayor receptividad. Cabe mencionar que en el segundo estadio, la reacción se limitó al ápice del estigma a comparación del estadio tres, donde la reacción fue en toda la superficie estigmática. En el primer estadio (preantesis) aparentemente no hay receptividad estigmática, sin embargo, la mayoría de las flores evaluadas mostraron indicios de estrés dejando la probabilidad que en esta etapa exista una receptividad muy baja. Si se confirma esta posibilidad, incrementaría aún más las oportunidades de polinización y fecundación (Balslev, 1996). El ovario del cuarto estadio (Fig. 2 D) tiene mayor tamaño que en los anteriores, lo que estaría indicando que los óvulos están fertilizados y estarían pasando por una etapa de crecimiento, por lo que el estigma tampoco sería receptivo en esta etapa. 


\section{P. Gonzáles et al. - Biología reproductiva y citogenética de Distichia muscoides}

Los géneros que forman almohadillados (Distichia, Oxychloe y Patosia) han desarrollado unisexualidad y dioecia como mecanismos de aislamiento. Las flores pistiladas se ocultan en las axilas de las hojas, y sólo los estigmas se proyectan por encima de las almohadillas. Las flores estaminadas se levantan en pedicelos largos por encima del almohadillado y están expuestos a polinizadores. En D. muscoides, un almohadillado constituye un clon y produce flores de un solo sexo. Almohadillados de sexos opuestos puede crecer muy cerca uno del otro, y las flores estaminadas y pistiladas pueden estar sólo a unos pocos centímetros de distancia o pueden estar separados por varios metros. Durante la floración, las flores están dispersas sobre toda la superficie del almohadillado. La gran mayoría de flores pistiladas son polinizadas, como lo demuestra la presencia de cápsulas con semillas (Fig. 2 A).

En cuanto al desarrollo del fruto, el ovario incrementa entre 2 a $3 \mathrm{~mm}$ de largo en un mes, duplicando su tamaño. Se estima que este proceso tardaría entre tres y cuatro meses hasta la maduración del fruto y posterior liberación de las semillas. Por lo general, se puede observar que cerca del $50 \%$ de los óvulos no llegan a ser fecundados. Sin embargo, esta disminución del éxito reproductivo se estaría mitigando con una producción masiva de flores y la una alta viabilidad de las semillas.

Las semillas de D. muscoides y $O$. andina Phil. son muy similares porque ambas presentan una cubierta reticulada, aunque $O$. andina tiene una reticulación regular a comparación de la superficie irregularmente reticulada de D. muscoides (Fig. $2 \mathrm{M}$ ). Esta similitud entre las semillas de las dos especies indica una estrecha relación y similares mecanismos de dispersión entre ellas (Balslev, 1996).

En los Andes, las temperaturas promedio suelen ser muy bajas, condición que podría influir en la temperatura óptima de germinación de semillas de plantas altoandinas. Este es el caso de la germinación de las semillas de Puya raimondi Harms, donde temperaturas mayores a $21^{\circ} \mathrm{C}$ la afectan negativamente (Berrie, 1984; Vadillo et al., 2004). Sin embargo, plantas con crecimiento almohadillado presentes en esta zona, como $D$. muscoides, tienen la capacidad de moderar la temperatura de su entorno y endógena (Salisbury \& Spomer, 1964; Arroyo et al., 1982; Nyakatya \& McGeoch, 2008), por lo que poseen temperaturas óptimas de germinación superiores. Esto podría explicar la causa del alto porcentaje de germinación de sus semillas a $28^{\circ} \mathrm{C}$. Asimismo, el almacenamiento por 18 meses a temperatura y humedad del ambiente $\left(21^{\circ} \mathrm{C}\right.$ y $80 \%$ en promedio) podría haber influido negativamente en la viabilidad de estas semillas, ya que estos factores conjuntamente con las condiciones de maduración, son importantes en el mantenimiento de la viabilidad de las mismas (Roberts 1972; Berrie, 1984; Soplin, 1988). El fotoperiodo considerado para esta evaluación constaba de $75 \%$ de horas luz y $25 \%$ de horas en la oscuridad, resultando en un alto porcentaje de germinación, el cual nos podría indicar indirectamente un efecto positivo de la luz en estas semillas; esto indicaría que aparentemente las semillas de D. muscoides son fotoblásticas positivas al igual que otras plantas altoandinas como Puya raimondi (Berrie 1984; Vadillo et al., 2004). Sin embargo, sería necesario evaluar su respuesta en oscuridad.

El periodo de germinación obtenido en el presente trabajo se encuentra entre los días 15 y 30 después de haber sembrado las semillas (tiempo promedio de germinación de 18,4 días), lo que podría permitir su establecimiento en la temporada húmeda en su hábitat.

Balslev (1996) sugirió que el agua podría actuar como vector de polen, sin embargo en el presente estudio no se encontraron diferencias significativas en la producción de semillas entre los frutos colectados bajo el nivel del agua y totalmente expuestos. Por ello concluimos que el principal o único vector de polen en $D$. muscoides es el viento, estando presente y con fuerte intensidad en su medio natural.

Las estrategias reproductivas como el desarrollo de largos pedicelos de las flores estaminadas que elevan las anteras y dejan expuesto el polen a los agentes dispersantes, la producción masiva de flores estaminadas y pistiladas, así como una buena exposición de las ramas estigmáticas con alta receptividad hacen más eficiente la polinización y fecundación. Otro aspecto importante es el desarrollo de un ginóforo que se alarga luego de la senescencia de la flor para compensar el corto pedicelo de las flores femeninas, levantándose por encima del almohadillado y exponiendo la cápsula madura para que las semillas puedan dispersarse libremente. Asimismo, la alta viabilidad 
de las semillas resulta ser otra estrategia para el mantenimiento de las poblaciones. Estas cualidades probablemente han hecho que esta especie logre alcanzar un gran éxito en su reproducción sexual, en comparación a las especies de sus géneros hermanos como $O$. andina Phil. y $P$. clandestina (Phil.) Buchenau, las cuales no presentan un ginóforo. En $O$. andina, las longitudes de los pedicelos de las flores pistiladas y estaminadas son similares (Balslev, 1996). Si a esto le sumamos la unisexualidad y la dioecia presentes en $D$. muscoides, se puede señalar que la reproducción sexual representa un mecanismo de éxito que le permite incrementar su variabilidad genética y asegurar el flujo de genes.

\section{Estudios cariológicos}

Se presenta por primera vez el número cromosómico de D. muscoides. Según la hipótesis filogenética de Záveská Drábková \& Vlček (2010), Distichia pertenece a un grupo monofilético, junto con especies de Juncus sec. Graminifolii, Marsippospermum Desv., Oxychloe y Patosia. En estos géneros, el número cromosómico básico varía entre $\mathrm{x}=8$ y $\mathrm{x}=20$, mientras que se ha reconstruido que el ancestro común del clado tiene $\mathrm{x}=20$ (Záveská Drábková, 2013). Según estos datos, además de que los conteos cromosómicos de este grupo usualmente son múltiplos de 20 (Záveská Drábková, 2013), y que nuestros análisis muestran la presencia de NORs en dos pares cromosómicos diferentes, se concluye que D. muscoides es tetraploide, y probablemente alopoliploide.

La distribución de diploides, agmatoploides, poliploides, agmatopoliploides, aneuploides y simploides es compleja dentro de Juncaceae, como también la distribución de los números cromosómicos sobre el árbol filogenético más reciente (Záveská Drábková, 2013), sumado a que el $58 \%$ de sus especies son cariológicamente desconocidas.

La presencia de cromosomas holocéntricos ha sido reportada tanto en Juncaceae como en Cyperaceae (Bozek et al., 2012; Bureš et al., 2013), siendo considerada una sinapomorfía para el clado constituido por ambas familias (Plunkett et al., 1995). Sin embargo, la presencia de esta clase de cromosomas solo ha podido ser demostrada satisfactoriamente en Luzula (Bureš et al., 2013). A diferencia de lo observado en este género, la existencia de constricción primaria permitió identificar cromosomas monocéntricos en Distichia. Sería necesario aplicar técnicas de citogenética molecular para mapear físicamente las secuencias centroméricas, con el fin de corroborar estos resultados. Por lo tanto, es preciso ampliar los estudios citogenéticos en cuanto a taxones y técnicas, para ser capaces de inferir tendencias evolutivas que ayuden a entender la diversificación de esta familia en un contexto biológico, ecológico y filogenético.

\section{Agradecimientos}

A Elluz Huamán y Warner Aparco por el apoyo en el trabajo de campo, a José Roque y Héctor Aponte por la revisión de manuscrito y sus importantes sugerencias, al Consejo Nacional de Investigaciones Científicas y Técnicas (CONICET) y al Instituto Multidisciplinario de Biología Vegetal por el apoyo a esta investigación.

\section{Bibliografía}

ARROYO, M. T. K., R. PRIMACK, \& J. ARMESTO. 1982. Community studies in pollination ecology in the high temperate Andes of central Chile. I. Pollination mechanisms and altitudinal variation. Amer. J. Bot. 69: 82-97.

BALSLEV, H. 1996. Juncaceae. Flora Neotropica 68: 1-167.

BERRIE, A M. M. 1984. Germination and dormancy. In: WILKINS, M. B. (ed.). Advanced plant physiology, pp. 440-468. The Pitman Press, Marshfield.

BOWEN, C. C. 1956. Freezing by liquid carbon dioxide in making slides permanent. Stain Technol. 31: 8790.

BOZEK, M., A. R. LEITCH, I. J. LEITCH, L. ZÁVESKÁ DRÁBKOVÁ \& E. KUTA. 2012. Chromosome and genome size variation in Luzula (Juncaceae), a genus with holocentric chromosomes. Bot. J. Linn. Soc. 170: 529-541.

BUREŠ, R. P., F. ZEDEK \& M. MARKOVÁ. 2013. Holocentric chromosomes. Springer, Vienna.

FAEGRI, K. \& J. IVERSEN. 1989. Textbook of pollen analysis. 4th ed. Blackburn Press, Caldwell.

FERREYRA, R. 1979. Sinopsis de la flora peruana, Gymnospermas y Monocotiledóneas. Los Pinos, Lima.

GALEN, C. \& R. C. PLOWRIGHT. 1987. Testing the accuracy of using peroxidase activity to indicate 


\section{P. Gonzáles et al. - Biología reproductiva y citogenética de Distichia muscoides}

stigma receptivity. Can. J. Bot. 65: 107-111.

HESSE, M., H. HALBRITTER, M. WEBER, R. BUCHNER, A. FROSCH-RADIVO \& S. ULRICH. 2009. Pollen Terminology: An illustrated handbook. Springer Science \& Business Media, Wien, New York.

HEUSSER, C. J. \& N. T. MOAR. 1973. Pollen and spores of Chile: Modern types of the Pteridophyta, Gymnospermae, and Angiospermae. New Zeal. J. Bot. 11: 389-391.

KIRSCHNER, J., H. BALSLEV, A. ČEŠKA, J. COFFEY SWAB, E. EDGAR, C. GARCÍA-HERRÁN, L. HÄMET-AHTI, Z. KAPLAN, L. J. NOVARA, V. S. NOVIKOV \& A. WILTON. 2002. Juncaceae 1: Rostkovia to Luzula. In: ORCHARD, A. E., J. BLEYERVEEN, A. J. G. WILSON \& B. KUCHLMAYR (eds). Species Plantarum: Flora of the World. Part 6: 1-237. Australian Biological Resources Study, Canberra.

NYAKATYA, M. \& M. MCGEOCH. 2008. Temperature variation across Marion Island associated with a keystone plant species (Azorella selago Hook. (Apiaceae)). Polar Biol. 3: 139-151.

PLUNKETT, G. M., D. E. SOLTIS, P. S. SOLTIS \& R. E. BROOKS. 1995. Phylogenetic relationships between Juncaceae and Cyperaceae: insights from rbcL sequence data. Amer. J. Bot. 82: 520-525.

RUTHSATZ, B. 1978. Las plantas en cojín de los semi-desiertos andinos del noroeste Argentino: Su distribución local como adaptación a los factores climáticos, edáficos y antropogénicos de sus ambientes. Darwiniana 21: 491-539.

SALISBURY, P. D. \& G. G.SPOMER. 1964. Leaf temperatures of alpine plants in the field. Planta 60: 497-505.

SCHWEIZER, D. 1980. Simultaneous fluorescent staining of $\mathrm{R}$ bands and specific heterochromatic regions (DA-DAPI bands) in human chromosomes. Cytogenet. Cell Genet. 27: 190-193.

SCHWEIZER, D. \& P. F. AMBROS. 1994. Chromosome banding. Stain combinations for specific regions. In: GOSDEN, J. R. (ed.). Methods in molecular biology 29. Chromosome analysis protocols, pp. 97-112. Humana Press, Totowa.
TORRES, G. R., L. C. LUPO, A. C. SÁNCHEZ, \& K. SCHITTEK. 2012. Aportes a la flora polínica de turberas altoandinas, Provincia de Jujuy, noroeste argentino. Gayana Bot. 69: 30-36.

TOVAR, O. 1973. Comunidades vegetales de la Reserva Nacional de Vicuñas de Pampas Galeras, Ayacucho, Perú. Publ. Mus. Hist. Nat. "Javier Prado" 27: 1-32.

VADILLO, G., M. SUNI, \& A. CANO. 2004. Viabilidad y germinación de semillas de Puya raimondii Harms (Bromeliaceae). Revista Per. Biol. 11: 71-78.

VALENCIA, N., A. CANO, A. DELGADO, H. TRINIDAD, \& P. GONZÁLES. 2013. Composición y cobertura de la vegetación de bofedales en un macrotransecto este-oeste en los Andes centrales del Perú. In: ALONSO, A., F. DALLMEIER \& G. SERVAT. (eds.). Monitoreo de Biodiversidad lecciones de un megaproyecto transandino, pp. 278-293. Smithsonian Institution Scholarly Press, Washington, DC.

WEBERBAUER, A. 1945. El mundo vegetal de los Andes peruanos. Lumen, Lima.

WILLE, J. N. F. 1882. Om pollenkornenes udvikling hos Juncaceer og Cyperaceer. Skr. Vidensk.-Selsk. Christiania, Math.-Naturvidensk. Kl. 16:1-4.

ZÁVESKÁ DRÁBKOVÁ, L. 2013. A Survey of Karyological Phenomena in the Juncaceae with Emphasis on Chromosome Number Variation and Evolution. Bot. Rev. 79: 401-446.

ZÁVESKÁ DRÁBKOVÁ, L \& Č. VLČEK. 2010. Molecular phylogeny of the genus Luzula DC. (Juncaceae, Monocotyledones) based on plastome and nuclear ribosomal regions: A case of incongruence, incomplete lineage sorting and hybridization. Molec. Phylog. Evol. 57: 536-551.

Recibido el 27 de agosto de 2015, aceptado el 14 de octubre de 2015. 
